\title{
Glycidyl Azide Polymer and Polyethylene Glycol as Hybrid Rocket Fuel
}

\author{
By Yutaka WADA ${ }^{1)}$, Keiichi HorI ${ }^{2)}$, Katsuya HASEGAWA ${ }^{2)}$, Tsuyoshi YAGISHITA ${ }^{2)}$, Kiyokazu KOBAYASHI ${ }^{2)}$, Shintaro \\ IWASAKI $^{2)}$, Hideki SATOH ${ }^{2)}$, Makito NISHIOKA ${ }^{3)}$ and Motoyasu KIMURA ${ }^{4)}$ \\ 1) Akita University, Akita, Japan \\ 2) Institute of Space and Astronautical Science, Japan Aerospace Exploration Agency, Sagamihara, Japan \\ 3) Tsukuba University, Tsukuba, Japan \\ 4) NOF Corporation, Aichi, Japan
}

(Received June 27th, 2011)

\begin{abstract}
Hybrid rockets that use glycidyl azide polymer (GAP) as a solid fuel were studied. The linear burning rate spectrum of GAP was broadened when it was diluted it with polyethylene glycol (PEG). Self-combustible mixtures were used for the gas hybrid rocket motor; non-self-combustible mixtures were used for the traditional hybrid motor. Both were tested as functions of various experimental parameters, and the results of the traditional hybrid rocket are summarized in this paper. In particular, the fuel regression rates of GAP50PEG50 and GAP60PEG40 fuels were investigated, and the results indicate that the fuel regression rate of the fuel with high GAP content showed pressure dependability. Simultaneous measurements of the surface regression rate, which has attracted great interest, were conducted using ultrasonics in a special chamber, the results for the instantaneous surface regression rate during combustion were obtained. The obtained results are presented, and on the basis of experimental observations, a flame model of the traditional hybrid motor is discussed.
\end{abstract}

Key Words: GAP, PEG, Hybrid Rocket, Ultrasonics

$\begin{array}{cl}\text { Nomenclature } \\ C_{p} & : \text { sonic speed of fuel grain } \\ G & : \text { mass flux } \\ r_{b} & : \text { surface regression rate } \\ P & : \text { pressure } \\ \rho & : \text { density } \\ \text { Subscripts } & \\ c & : \text { chamber } \\ o x & : \text { oxygen } \\ f & : \text { fuel }\end{array}$

\section{Introduction}

Glycidyl azide polymer (GAP) is one of the most promising high energy materials for the future because it exhibits high heat of formation, high density, and low hazard. Use of GAP as a fuel binder in composite propellants, solid fuels of hybrid rockets, and ducted rockets has been widely investigated ${ }^{1-9)}$. Owing to its self-combustibility, GAP is a promising solid fuel in gas generator (GG)-type hybrid rocket systems, which are called gas hybrid rockets ${ }^{10,11)}$. Attempts have been made to broaden the burning rate spectrum of GAP, which is the range of various burning rates, in order to widen the design scope of motors from those powering small sounding rockets to large boosters. GAP burns at higher rates $(15 \mathrm{~mm} / \mathrm{s} @ 5 \mathrm{MPa})$ as compared to conventional composite propellants; therefore, it is necessary to suppress the burning rate of GAP to a considerable extent, as required. In general, it is desirable to have a burning rate of several $\mathrm{mm} / \mathrm{s}$ for small diameter rockets such as sounding rockets to $15-20 \mathrm{~mm} / \mathrm{s}$ for large boosters. Among several methods, the addition of an inert polymer whose solubility is close to GAP was found to be the only feasible method to increase the burning rate. Several polymers were investigated, and polyethylene glycol (PEG) was selected owing to its good solubility in GAP, use of the same curing system, favorable density $\left(1.17 \times 10^{3} \mathrm{~kg} / \mathrm{m}^{3}\right)$, and low cost. With the addition of PEG, the burning rate is easily suppressed while the pressure exponent is kept constant and the mechanical property of the mixture is improved ${ }^{11)}$. The resulting burning rate spectrum is wide enough to cover the entire desired range, from that for small sounding rockets to the largest boosters. The combustible limit of the mixture in terms of PEG concentration is $50 \mathrm{wt} \%$; further addition of PEG makes the mixture noncombustible. Combustible mixtures have been used in gas hybrid rockets, and noncombustible mixtures containing more than $50 \%$ of PEG have been used in the traditional hybrid rockets in a previous study $^{11)}$.

Dilution of GAP with PEG lowers the energy content of the solid fuel itself and, therefore, the specific impulse (Isp) of the hybrid system. The density specific impulse ( $\rho I s p)$ of various fuels with different concentrations of GAP and PEG are compared in Fig.1. Here, the simple product of the density of the fuel $\left(\rho_{f}\right)$ and the $I s p$ is used for comparison, and the number in the name of mixtures corresponds to the mass fraction of each polymer. The high density of GAP is one of its advantages, as a solid fuel, over hydroxyl-terminated polybutadiene (HTPB), and PEG's density is also higher than that of HTPB. Therefore, GAP and GAP+PEG fuels can enable the design of compact chemical propulsion systems. A comparison with HTPB is also made, and owing to the high density of GAP $\left(1.3 \times 10^{3} \mathrm{~kg} / \mathrm{m}^{3}\right)$, medium density of PEG, 
and low density of HTPB $\left(0.9 \times 10^{3} \mathrm{~kg} / \mathrm{m}^{3}\right)$, the difference in $\left(\rho_{f}\right) \times I s p$ of the mixtures of GAP, PEG, and HTPB can be clearly observed in Fig.1; even pure PEG is noticeably superior to HTPB.

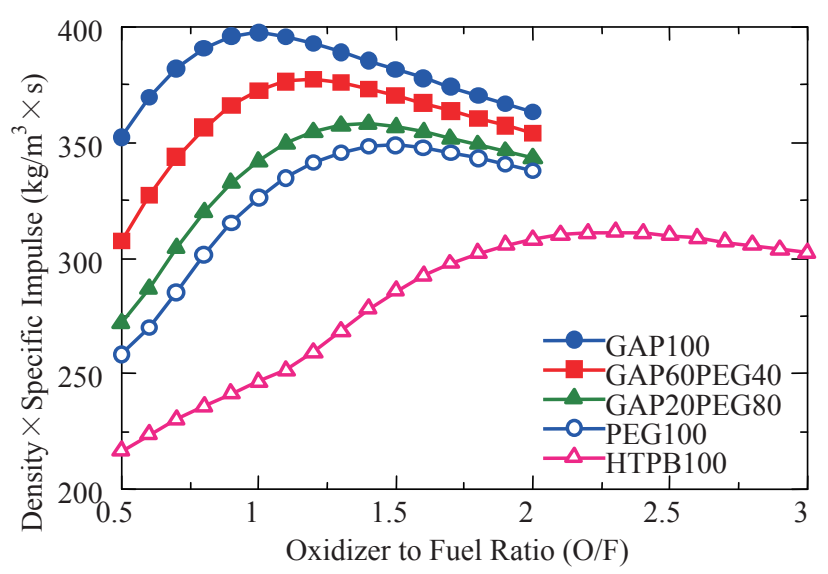

Fig. 1. Comparison of $\rho_{f} \times I s p$. (Pc: $5 \mathrm{MPa}$; Expansion: Optimum in vacuum)

Traditional firing tests were conducted using gaseous oxygen $\left(G_{o x}\right)$ with a sub-scale-sized test motor (inner diameter $=80 \mathrm{~mm}$ ). Many studies have been conducted on this type of hybrid rocket using an inert polymer as a solid fuel, and the regression rates of the solid fuel grain have been found to be less than satisfactory ${ }^{12,13)}$. Recently, wax with additives such as nano-aluminum and aluminum hydride has been recognized as a solid fuel with a high regression rate ${ }^{12,14)}$; however, its poor mechanical properties and adhesion with other materials may hamper the application of this material to full-sized rocket motors. A mixture of GAP and PEG, however, may offer a solution to these problems because of the high energy, excellent mechanical properties, and hydroxyl terminated structure of GAP, which makes it possible to employ the same technology for solid rocket motor manufacturing with HTPB based propellants. The burning test was started from the minimum oxygen flow rate and burning pressure from the viewpoint of safety and, therefore, the data recorded was limited; however, the surface regression rates $\left(r_{b}\right)$ of GAP/PEG mixtures are significantly higher than that of inert polymers as reported earlier ${ }^{11}$. In this report, both oxygen flow rate and burning pressure were increased, and the GAP60PEG40 sample, which is combustible and is categorized as a fuel for gas hybrid motors, was employed in a traditional hybrid motor. The dependencies of $r_{b}$ on oxygen flow rate, burning pressure, and equivalent ratio were analyzed by principal component analysis and multiple regression analysis, and the results were used to develop a combustion model of these mixtures in a traditional hybrid rocket motor.

Surface regression rates generally have a mean value, and in this study, these were obtained typically by conducting the burning tests for $10 \mathrm{~s}$. However, every experimental parameter in traditional hybrid motors changes gradually as the combustion proceeds; thus, the measurement of the instantaneous $r_{b}$ is very much necessary for the detailed discussions. The ultrasonic measurement technique developed at ONERA ${ }^{15,16)}$ used to evaluate the burning rates of full-scale solid rocket motors in Japan ${ }^{17)}$ was employed in this study. The combustor and ultrasonic sensors were specially designed for this purpose. Three ultrasonic sensors located along the $1300 \mathrm{~mm}$ length chamber at the start, middle, and end positions can be excited up to $100 \mathrm{~V}$, which enables signal acquisition from the regressing fuel surface on the experimental scale used in this study. Moreover, special software developed for this study was used to deduce the signal from the burning surface at $100 \mathrm{~Hz}$. Burning tests were started with a PEG100 sample, and the local and instantaneous surface regression rates were successfully obtained.

\section{Experimental}

\subsection{Fuel sample}

Tetra-ol GAP developed by NOF has been used. No crosslinking agent is required to cure this polymer; however, PEG is a di-ol and needs a crosslinking agent. Trimethylolpropane (TMP) was used as the crosslinking agent, and hexamethylenediisocyanate (HMDI) and dibutyltindilaurate (DBTDL) were used as the curing agent and the catalyst for the curing reaction, respectively. The fuel mixture contains GAP and PEG in a specified ratio, and the TMP content is proportional to the PEG concentration. HMDI was added with a concentration equal to the sum of GAP and PEG; and a trace amount of DBTDL was added. Mixing and casting must be done at a temperature higher than the melting temperature of PEG to prevent its precipitation. A thorough evacuation was performed during mixing and casting to eliminate bubbles from the fuel grain. All the processes were successfully conducted at the ISAS laboratory.

In the past study, non-self-combustible mixtures (PEG100 to GAP50PEG50) were used in the traditional hybrid system; however, in this study, a GAP60PEG40 sample is employed in the system to achieve a higher surface regression rate.

\subsection{Hybrid rocket testing}

GAP/PEG mixtures were loaded in a polyvinyl chloride (PVC) tube as a simple internal burning tube grain (Fig.2), and both its ends were blocked with an inert polymer. A schematic of the experimental setup is shown in Fig.3. The chamber length was adjustable to four levels: $95 \mathrm{~mm}, 242 \mathrm{~mm}$ (Fig.3), $400 \mathrm{~mm}$, and $600 \mathrm{~mm}$. Oxygen gas was introduced into the combustion chamber through four ports, then forced to flow along the wall of the pre-combustion section, and finally converged into the bore of the grain. The same igniter was used as that used in the firing tests of solid motors, and it is composed of an initiator and non-metallized solid propellant grain, and the oxygen flow was started $1 \mathrm{~s}$ before ignition. Figure 4 is the photograph of the experimental setup of the 
hybrid test motor. Typically, ten seconds burning tests were conducted, and the mean values of the surface regression rate, burning pressure, oxygen mass flux $\left(G_{o x}\right)$, and $\mathrm{O} / \mathrm{F}$ (oxidizer to fuel ratio) were evaluated. $G_{o x}$ was calculated from the cross-sectional area of the fuel tube. The inner diameter increases during combustion, and thus, the mean value means the average of the initial and the final values of the inner diameter.

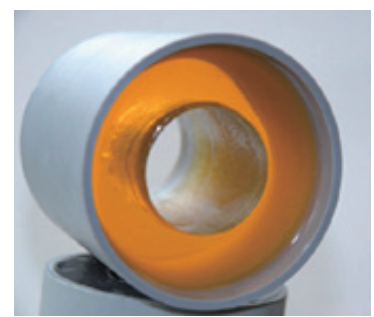

Fig. 2. Sample grain. (Diameter: $85 \mathrm{~mm}$, Inner Diameter: $40 \mathrm{~mm}$ )

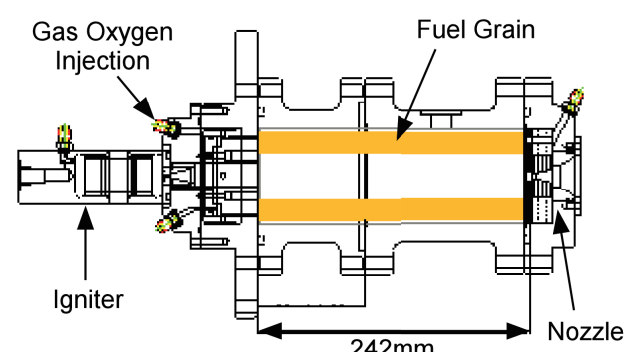

Fig. 3. Hybrid test motor.

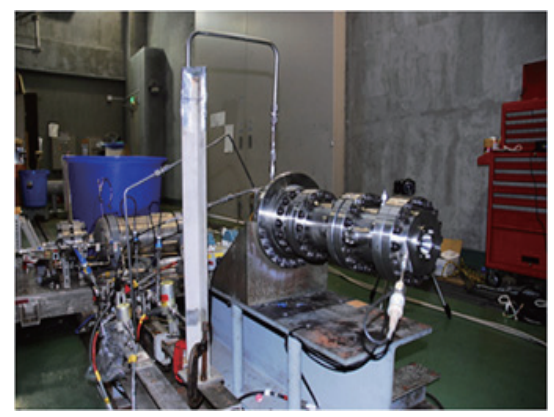

Fig. 4. Setup of hybrid test motor.

\subsection{Ultrasonic measurement}

A special combustor was developed for this purpose. Its inner diameter and length are $80 \mathrm{~mm}$ and $1300 \mathrm{~mm}$, respectively, and the chamber is equipped with three ultrasonic measurement ports and three temperature measurement ports (Fig.5). An ultrasonic sensor was also

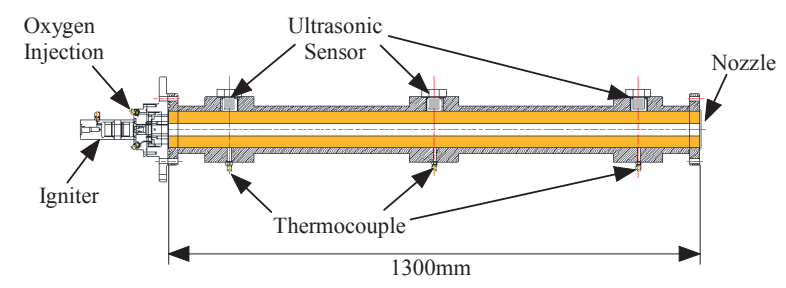

Fig. 5. Hybrid test motor for ultrasonic measurement. specially designed and manufactured by Honda Electronics Co. Ltd. The sensor diameter is $20 \mathrm{~mm}$ and it is composed of a set of a pulser and a receiver as shown in Fig.6. The wave frequency was set at $1 \mathrm{MHz}$ because an ultrasonic wave of this frequency penetrates rubber materials well and is suitable for this measurement. The high power ultrasonic generator, OmniScan MX (Olympus Co.) was used for the ultrasonic wave generation and data recording. A stainless holder was set at the ultrasonic measurement port and the sensor was attached to it applying grease to maintain good contact. The fuel mixture was cast directly in the chamber at a simple internal burning tube grain and the adhesion between the holder and fuel grain was good enough.

Ultrasonic measurement requires the calibration of sonic speed, $C_{p}$, within the sample as a function of pressure and temperature, because the instantaneous surface regression rate is obtained by taking the time derivative of the trace of the fuel grain thickness, and the instantaneous fuel web thickness is obtained using the traverse time of the ultrasonic signal from the regressing surface and the $C_{p}$ at the instantaneous burning pressure and grain temperature. The $C_{p}$ calibration of each fuel polymer was done independent of the burning tests as functions of pressure and temperature using a specially designed device.

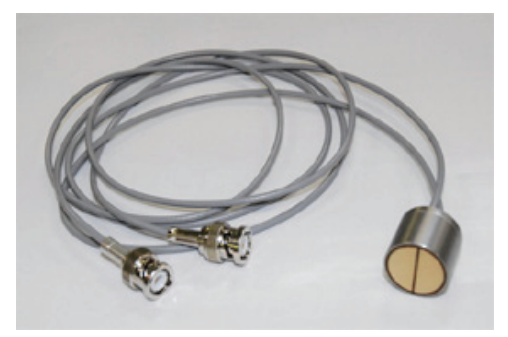

Fig. 6. Ultrasonic sensor.

\section{Results and Discussion}

\subsection{Surface regression rate: $r_{b}$}

Ten seconds burning tests were conducted and $r_{b}$ was evaluated as a mean value. Figure 7 shows the hybrid firing test of the GAP60PEG40 sample.

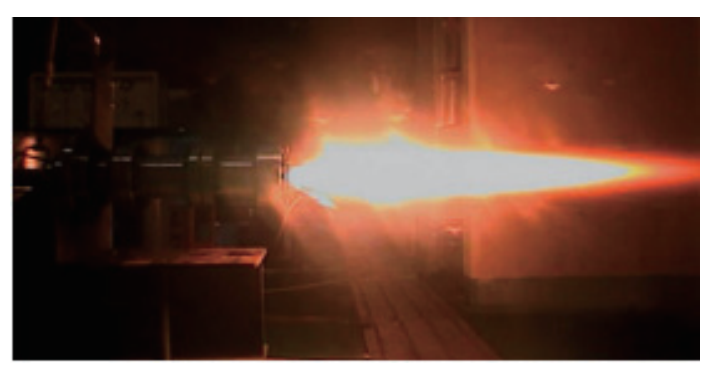

Fig. 7. Firing test of GAP60PEG40.

All the results, including previous data, are summarized in Figs. 8 and 9 as functions of GAP concentration, $G_{o x}$, and burning pressure. As shown in Figs. 8 and 9, all the three 
parameters have a positive effect on $r_{b}$; however, the dependence of $r_{b}$ on $G_{o x}$ and pressure cannot be evaluated independently from these two graphs because they have a mutual effect and other parameters may contribute to $r_{b}$.

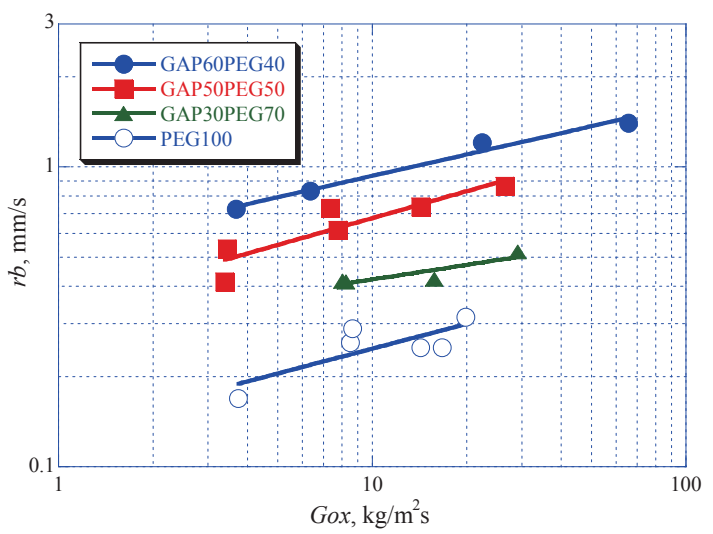

Fig. 8. Surface regression rate as a function of $\mathrm{G}_{o x}$.

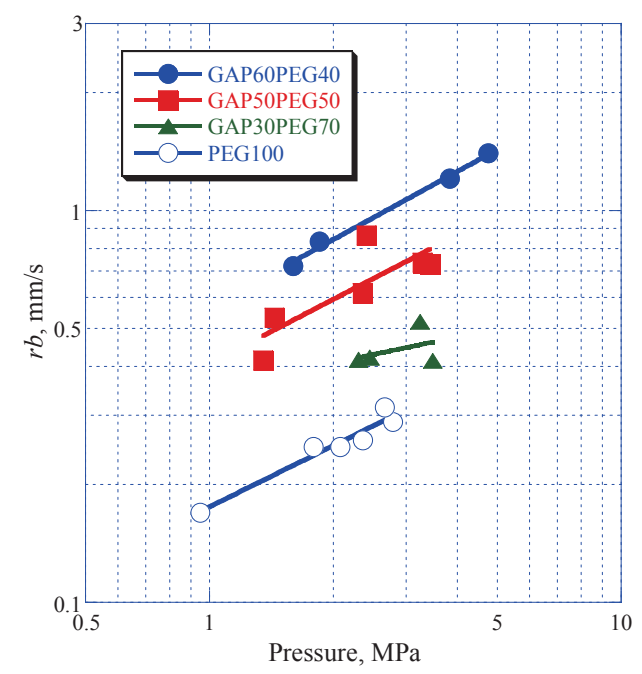

Fig. 9. Surface regression rate as a function of pressure.

Therefore, attempts were made to estimate the dependencies of $r_{b}$ on the parameters $G_{o x}$, pressure, and $O / F$ at each fuel mixture using principal component analysis and multiple regression analysis in spite of the small amount of data and relatively large data fluctuation. First, principal component analysis was used to understand the strength of the effect of each parameter qualitatively. From this analysis, it was found that 1) the dependency on $G_{o x}$ is always recognized, 2) the higher the GAP concentration, the greater the dependency on burning pressure, and 3) the higher the GAP concentration, the lower the dependency on $O / F$. 1) is naturally understood because the heat input from the main gas flow to the regressing surface is always important for $r_{b}$, and 2) and 3) seem to be consistent because GAP has self-combustibility, a high burning rate, and a pressure exponent; thus, $r_{b}$ of the mixtures with higher GAP concentration may be affected by the combustion of GAP and may have a pressure exponent. On the other hand, the effect of GAP may be small for the mixtures with lower GAP concentration and their $r_{b}$ may be affected by the strength of the diffusion flame between the fuel gas from PEG and oxygen.

At the next step, taking account of these results, multiple regression analysis was employed to obtain the quantitative relationship between $r_{b}$ and the experimental parameters. Multi-regression analysis was used to calculate "a" factor, "n" power, and " $m$ " power of equation (1). We take the logarithm of equation (1) as shown in equation (2), and substitute each $\log a$, " $n$," and " $m$ " for each corresponding term in equation (3). Equation (3) is the multiple regression equation. Log a, "n," and "m" in equation (2) were calculated using regression analysis.

$$
\begin{gathered}
r_{b}=a G_{o x}^{n} P_{c}^{m} \\
\log r_{b}=\log a+n \log G_{o x}+m \log P_{c} \\
y=a+b_{1} x_{1}+b_{2} x_{2}+\cdots
\end{gathered}
$$

As can be seen in Fig.9, the comparison between GAP50PEG50 and GAP60PEG40 is adequate for the discussion on the burning pressure effect; further, this comparison is important because the self-combustibility limit of GAP and PEG mixtures lies between these two concentrations.

For the GAP50PEG50 sample, rb can be expressed as

$$
r_{b}=0.34 G_{o x}^{0.20} P_{c}^{0.23},
$$

and for the GAP60PEG40 sample

$$
\begin{gathered}
r_{b}=0.56 G_{o x}^{0.06} P_{c}^{0.41} \cdot \\
\left(r_{b}: \mathrm{mm} / \mathrm{s}, \quad G_{o x}: \mathrm{kg} / \mathrm{m}^{2} \cdot \mathrm{s}, \quad P_{c}: M P a\right)
\end{gathered}
$$

From the comparison of these two equations, $50-60 \mathrm{wt} \%$ of GAP is the range where the combustion mechanism changes. At $60 \mathrm{wt} \% \mathrm{GAP}, r_{b}$ seems to be dominated by the combustion of GAP itself because the pressure exponent of the self-burning rate of GAP is approximately 0.4 in this pressure region. Moreover, it can be predicted that further addition of GAP may enhance the pressure dependency, and at some critical concentration, the mixture becomes unacceptable for rocket motor application because of an extremely high pressure exponent.

As reported in the earlier paper ${ }^{18)}$, the quenched surface of PEG100 is covered with a transparent PEG melt layer, and GAP50PEG50 is dry, dark blown colored, and covered with combustion residue. Figure 10 shows the quenched surface of the GAP60PEG40 sample. The color of the surface is closer to black and this may show that the GAP site at the regressing surface is covered with soot, which is also observed at the burning surface of GAP itself ${ }^{19), 20)}$. 


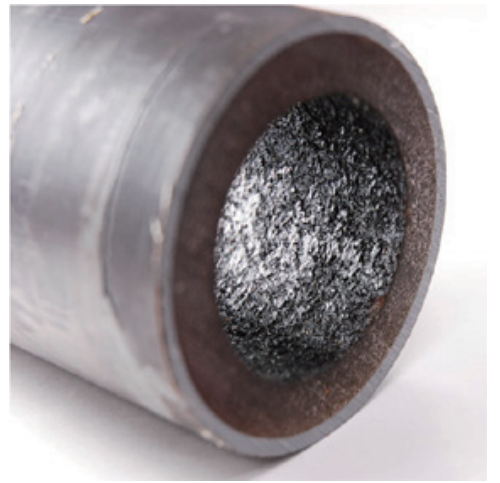

Fig. 10. Quenched surface of GAP60PEG40.

Figure 11 shows our flame model of GAP/PEG mixtures in a traditional hybrid rocket motor. In the case of PEG100 (Fig.11 (a)), the polymer surface is covered with PEG melt and droplets may depart from the surface or large molecules may sublimate into the gas phase. The degradation process of large fuel molecules is relatively slow and the standoff distance of the flame sheet is large, and the lower heat feedback to the surface retards the surface regression rate. On the other hand, in the case of the mixture of GAP and PEG, GAP burns at its site on the regressing surface, and large heat is released due to the decomposition of azide groups, which makes the degradation of fuel molecules easier and the standoff distance of the flame with respect to the GAP site shorter. The self-combustion of GAP induces the pressure-driven nature of the $r_{b}$ of this mixture and the large heat released may affect the behavior of PEG. The surface temperature of the PEG site as well as the vaporization rate may be enhanced, and fuel vapors may degrade faster than pure PEG and the flame standoff distance may become shorter. A distributed diffusion flame may be formed, as shown in Fig.11 (b), closer than the flame sheet of pure PEG (Fig.11 (a)), which may cause $r_{b}$ enhancement. Further, in the case of GAP combustion, the energetic popping-out motion of the released nitrogen gases in the surface melt layer promotes the departure of GAP melt droplets from the burning surface ${ }^{19), 20)}$, and this may also occur at the regressing surface in the hybrid motor. Droplets of PEG melt may be forced to depart from the regressing surface with this popping-out motion, which can be another reason for the higher $r_{b}$ of GAP and PEG mixtures.

To Injector

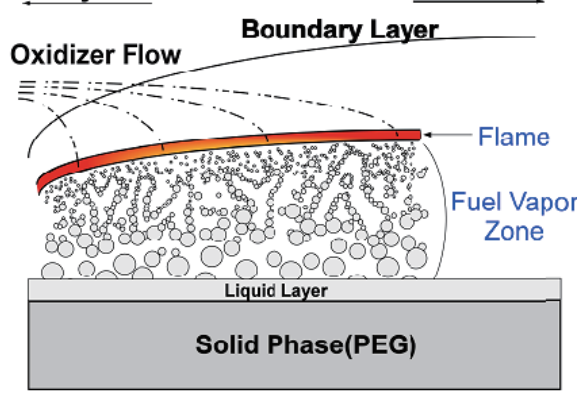

(a) PEG100 sample.

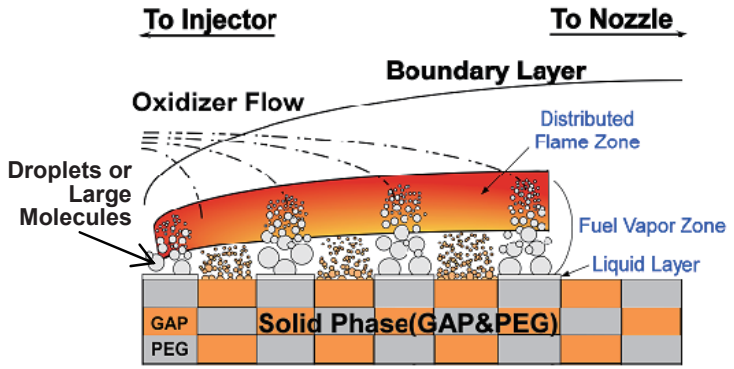

(b) GAP50PEG50 sample.

Fig. 11 Flame model of traditional hybrid rocket motor.

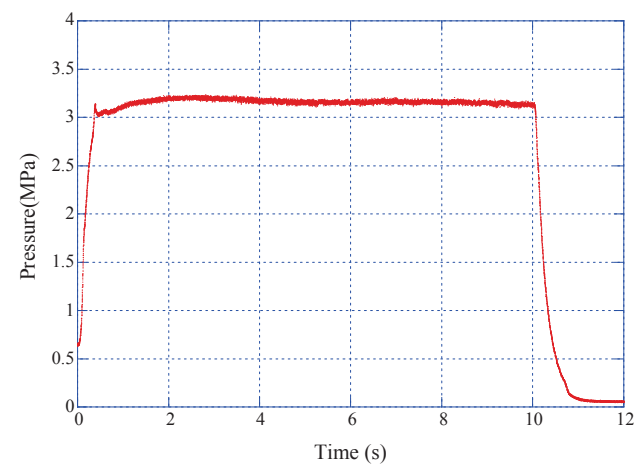

Fig.12. Pressure pattern of a hybrid burn test. (Fuel: PEG100, $G_{o x}: 78 \mathrm{~kg} / \mathrm{m}^{2} \mathrm{~s}$ ).

\subsection{Ultrasonic measurement}

Burning tests were conducted using PEG100. Strong multi-reflection within the stainless steel holder plate and the signal through the peripheral part of the combustor hampered the ultrasonic measurement; however, specially developed software makes it possible to pick up the signal from the regressing surface with an acceptable accuracy. Figure 12 shows an example of the pressure pattern of the hybrid test.

Due to the low regression rate of PEG, a nearly constant pressure pattern was observed for the ten seconds burn. Figure 13 is the thickness trace of the burnt PEG fuel, which is obtained from the traverse time of the ultrasonic signal and the sonic speed of the PEG as a function of pressure and initial temperature of the grain. Instantaneous surface regression rate is obtained as the time derivative of this curve as shown in Fig.14. For example, the regression rate at $2.0 \mathrm{~s}$ reduced from $0.46 \mathrm{~mm} / \mathrm{s}$ to $0.28 \mathrm{~mm} / \mathrm{s}$ at $8.0 \mathrm{~s}$, which shows that the regression rate is higher at the initial stage because of smaller bore area and therefore, the $G_{o x}$ is higher.

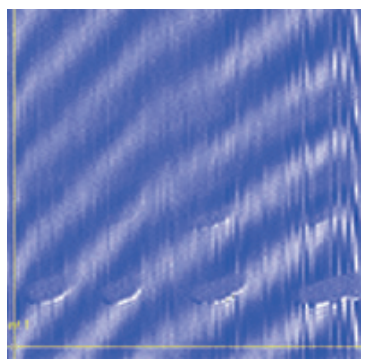

Fig. 13. Trace of fuel burnt. (Fuel: PEG100, $G_{o x}: 78 \mathrm{~kg} / \mathrm{m}^{2} \mathrm{~s}$, Pc: 3 $\mathrm{MPa})$. 
can be effectively used to obtain the instantaneous regression rate, and such data is useful to appropriately discuss the effects of the experimental parameters on the regression rate; thus, the ultrasonic technique is indispensable in the establishment of the combustion mechanism of hybrid rocket.

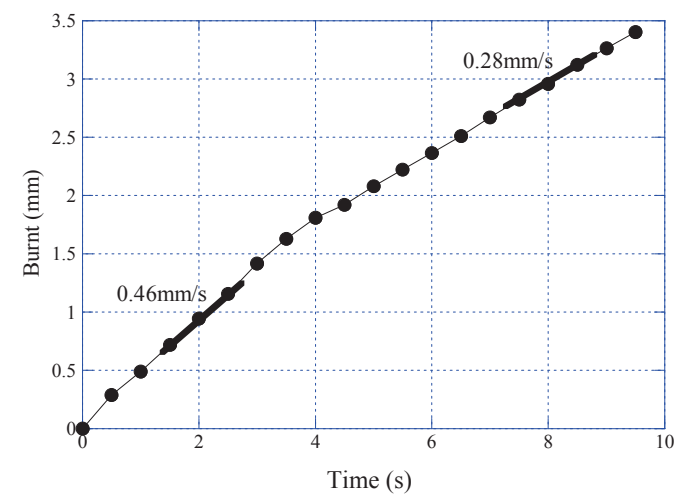

Fig. 14. Data reduction of instantaneous surface regression rate. (Fuel: PEG100, $G_{o x}: 78 \mathrm{~kg} / \mathrm{m}^{2} \mathrm{~s}$, Pc: $3 \mathrm{MPa}$ ).

\section{Summary}

Mixtures of GAP and PEG from PEG100 to GAP60PEG40 were tested in a traditional hybrid rocket system using gaseous oxygen as an oxidizer with a small test motor. The fuels get extinguished when the oxygen is cut off and the versatility of quench and the re-ignition of hybrid rocket motor were found to be maintained for all the samples. Surface regression rates were summarized, and it was found that when GAP concentration is higher, the surface regression rate becomes higher. Surface regression rates of all the mixtures show positive dependencies on $G_{o x}$ and $P_{c}$. Principal component analysis and multiple regression analysis were employed to evaluate the effect of each experimental parameter, and the burning pressure dependency was found to be stronger and the $G_{o x}$ dependency weaker for higher GAP concentration samples.

The ultrasonic technique was employed in the hybrid motor, and a special sensor was developed for this purpose. A trial was conducted to measure the regression rate of the PEG fuel.

The time history of the traverse time of the ultrasonic signal from the regressing surface was successfully measured, and the instantaneous regression rate was obtained using the calibration data of the ultrasonic speed of PEG as a function of pressure and temperature. This measurement will help greatly in establishing a comprehensive combustion model of hybrid rocket motors.

\section{Acknowledgments}

This research was supported by the Hybrid Rocket Research Working Group (HRrWG) of the Institute of Space and Astronautical Science, Japan Aerospace Exploration
Agency. The authors thank the members of HRrWG for the helpful discussion.

\section{References}

1) Aiello, P.F. and Manzara, P.: GAP Benefits, JANAAF Combustion Meeting (1984), p.399.

2) Davidson, J.E. and Becketead, M.W.: A Mechanism and Model for GAP Combustion, AIAA Paper (1997), pp.97-0592.

3) Frankel, M.B., Grant, L.R. and Flanagan, J.E.: Historical Development of Glycidyl Azide Polymer, J. of Propulsion and Power, 8 (1992), p.560.

4) Helmy, A.M.: GAP Propellant for GAS Generator Application, AIAA Paper (1987), pp.87-1275.

5) Hori, K. and Kimura, M.: Combustion Mechanism of Glycidyl Azide Polymer, Propellants, Explosives, Pyrotechnics, 21 (1996), p. 160 .

6) Kato, K. et al.: Mechanical Properties of GAP Reduced Smoke Propellants, AIAA Paper (1996), pp.96-3253.

7) Korobenichev, O.P. et al.: GAP Decomposition and Combustion Chemistry Studied by Molecular Beam Spectroscopy, AIAA Paper (1999), pp.99-0596.

8) Kubota, N. and Sonobe, T.: Combustion Mechanism of Azide Polymer, Propellants, Explosives, Pyrotechnics, 13 (1988), p.192.

9) Schoeyer, H.F. et al.: First Experimental Results of an HNF/Al/GAP Solid Rocket Propellant, AIAA Paper (1997), pp.97-3131.

10) Wada, Y. et al.: Combustion Mechanism of tetra-ol Glycidyl Azide Polymer and its Application to Hybrid Rockets, Proceedings of 7-ISICP (2008), pp.1099-1114.

11) Hori, K.: Application of Glycidyl Azide Polymer to Hybrid Rocket Motor, AIAA Paper (2009), pp.2009-5348.

12) Chiaverini, M. and Kuo, K.K., Eds.: Fundamentals of Hybrid Rocket Combustion and Propulsion, Progress in Astronautics and Aeronautics (2006), p.218.

13) Deluca, L. and Rossettini, L.: Ballistic Characterization of AlH3-Based Propellants for Solid and Hybrid Rocket Propulsion, AIAA Paper (2009), pp.2009-4874.

14) Evans, B., Boyer, E., Kuo, K.K., Risha, G. and Chiaverini, M.: Hybrid Rocket Investigations at Penn State University's High Pressure Combustion Laboratory: Overview and Recent Results, AIAA Paper (2009), pp.2009-5349.

15) Cauty, F. and Demarais, J.C.: Ultrasonic Measurement of the Uncured Solid Propellant Burning Rate, Proceedings of $21 \mathrm{st}$ International Conference of ICT, 1990.

16) Cauty, F.: Electronic Device for Ultrasonic Measurements (EDUM) of Regression Rates of Solid Materials, ONERA Report, 1995.

17) Kurabayashi, H. et al.: Ultrasonic Measurements of Burning Rates in Full-size Rocket Motors, Proceedings of 3-EUCASS, printing.

18) Hori, K., Nomura, Y., Fujisato, K., Yagishita, T., Hasegawa, K., Kobayashi, K., Nishioka, M., Wada, Y. and Kimura, M.: Hybrid rocket using GAP as a solid fuel, presented at 8-ISICP, IJEMCP, in press.

19) Wada, Y., Seike, Y., Tsuboi, N., Hasegawa, K., Kobayashi, K., Nishioka, M. and Hori, K.: Combustion mechanism of tetra-ol glycidyl azide polymer, Science and Technology of Energetic Materials, 69 (2008), pp.143-148.

20) Wada, Y., Seike, Y., Tsuboi, N., Hasegawa, K., Kobayashi, K., Nishioka, M. and Hori, K.: Combustion model of tetra-ol glycidyl azide polymer, Proceedings of the Combustion Institute, 32 (2009), pp.2005-2012. 